\title{
An Appraisal of Novel Biomarkers for Evaluating and Monitoring Neurologic Diseases: Editorial Introduction
}

\author{
Jeremy M. Shefner ${ }^{1} \cdot$ Marwan N. Sabbagh ${ }^{1}$
}

Published online: 8 December 2016

(C) The American Society for Experimental NeuroTherapeutics, Inc. 2016

Neurology as a medical field has evolved from the twin disciplines of clinical observation and pathology. The earliest descriptions of diseases were purely phenomenologic, with elegant descriptions of seizures, strokes, and movement disorders. Pathology contributed to knowledge very early as well, with Charcot's description of the lateral spinal cord appearance in amyotrophic lateral sclerosis (ALS), and descriptions of multiple sclerosis (MS) plaques among many others [1]. In the nineteenth century, Helmholtz and others investigated the physiological properties of both normal and diseased nerve, heralding the development of clinical neurophysiology [2]. More recently, dramatic improvement in imaging modalities has allowed for specific diagnoses, and biofluid evaluations have become an integral part of routine medical practice.

The development of imaging, biochemical, physiologic, and cell-based evaluation has revolutionized the practice of medicine. With respect to clinical therapeutic development, however, until recently evaluation of new therapies has been largely dependent on extensions of clinical observation. Migraine studies use headache diaries, Epilepsy studies use seizure diaries, and clinical trials of neurodegenerative and neuromuscular diseases have largely depended on clinical activities such as timed up-and-go tests, a 6-min walk, and other reflections of clinical status. While such evaluations have led to significant therapeutic advances, they have limited ability to discern pathophysiologic pathways. Thankfully, as our

Jeremy M. Shefner

Jeremy.Shefner@DignityHealth.org

Marwan N. Sabbagh

marwan.sabbagh@dignityhealth.org

1 Department of Neurology, Barrow Neurological Institute, Phoenix, AZ, USA understanding of normal and abnormal human biology has advanced, so have tools for assessing disease pathways. The reviews in this issue of Neurotherapeutics assess the ways in which improved technology has merged with deeper understanding of neurologic disease to yield a spectrum of markers that will, without question, speed the development of new therapies in neurology.

The markers discussed in this issue revolve around 4 general modalities: imaging, physiology, function, and biofluids. Advances in imaging have led to an increased ability to discern structural changes that make quantitative imaging a powerful outcome measure [3-5]. In ALS, quantitative magnetic resonance imaging (MRI) technology can show abnormalities prior to the development of symptoms, and clear changes occur as the disease progresses [4]. It is as yet unknown whether the rate and variability of such changes can increase trial efficiency compared with the commonly used functional outcome measures, which currently are the mainstay of most ALS trials. Standardization of imaging techniques across clinical trial sites is an added challenge, as is overall expense. MS trials have used MRI for years to assess disease activity. For the most part, however, MRI is much more sensitive to white matter lesions, which underlie progression in relapsingremitting MS than to the gray matter disease that is thought to be the basis of disability in primary and secondary progressive MS [5]. In this case, the presence of an imaging marker correlates well with the development of therapies, which have been sparse in progressive MS. New MRI modalities are also beingdevelopedtoaidinseizurefocusidentificationwiththegoal of improved surgical precision in patients with epilepsy; when combined with magnetoencephalography, focus localization is improvedfurther[3].

Beyond the improvements in structural identification, functional MRI, as well as positron emission tomography and single-photon emission computed tomography, can identify 
and follow neural networks, localize abnormal protein deposits, and localize specific receptors and their binding to potential therapeutic agents. Tau and amyloid are being imaged in ongoing clinical trials in Alzheimer's disease (AD) [6-8]; it remains to be seen whether changes in protein burden correlate well with changes in clinical phenotype. However, such imaging studies have already enhanced our understanding of the distribution of disease burden in patients with $\mathrm{AD}$, as well as the potential for therapeutic agents to ameliorate accumulation. Fludeoxyglucose positron emission tomography has also been used to assay pathway integrity in $\mathrm{AD}$; it is still unknown whether sequential studies can measure disease progression more sensitively than currently used techniques [6].

Clinical neurophysiology has aided in the diagnosis of peripheral neuromuscular diseases for many years; however, electromyogram/nerve conduction studies have been of limited use in detecting either disease progression or response to treatment, with the exception of demyelinating neuropathies. In 1971, McComas et al. [9] proposed a method for estimating the number of motor units innervating a muscle being studied; such a method would clearly be of great utility in following disease progression in motor neuron diseases or other axonal injuries. However, issues surrounding inter-rater reliability and validity rendered the original method and subsequent alternatives less useful than originally thought. More recently, however, interest in estimating motor unit numbers has been revived, and new methods have been proposed that address many of the limitations of prior methods. Motor unit estimation with a variety of new techniques are currently being employed in clinical trials in ALS and spinal muscular atrophy, and hold promise to detect sensitively a response to therapy [10]. Other techniques have also been developed to query axon function. Intramembrane processes such as ionconductance channels can be studied using a technique developed for use in humans as an extension of the threshold electrotonus technique developed by Hugh Bostock. These methods can specifically evaluate axonal excitability changes owing to alterations in slow and fast conductance channels specific to a variety of ions, and have shown abnormalities in ALS, diabetic neuropathy, and other peripheral nerve processes [11]. Given that abnormal excitability in axons and cell bodies has been suggested to be important in ALS, these techniques are currently being utilized in several clinical trials studying drugs expected to affect ion channels. Similar techniques are being used in motor cortex, where transcranial magnetic stimulation paradigms have been developed to evaluate corticomotor neuron excitability [12]. Finally, magnetoencephalography combined with complex imaging techniques is now assisting surgeons in efficiently planning epilepsy surgery [3].

While bioimpedence measures have been used for many years to evaluate processes as diverse as body fat and geologic structures, it has only recently been applied to skeletal muscle
[13]. Electrical impedance myography (EIM) has been shown to distinguish between neurogenic and myogenic muscle pathology, and preliminary studies have suggested that EIM is a more sensitive measure of disease progression than other outcomes used in ALS trials. While changes in EIM are not related to disability in a simple way, the fact that changes occur rapidly and reproducibly make this measure very attractive in phase II trials.

With respect to fluid markers, rapid developments are occurring in ALS, AD, and inflammatory neurological diseases $[6,7,14,15]$. In ALS, markers under current development probably reflect cell death or response to stress rather than specific disease pathways. Cell damage markers such as neurofilament in blood and cerebrospinal fluid may closely match other measures of disease progression, with reliability that may allow for a reduction in trial size in phase II trials. Blood urate, which increases in a large number of neurodegenerative diseases, may also be a marker for improved outcome; as urate has antioxidant properties, it is possible that urate levels may be a modifiable target for intervention as well [16]. In contrast, $A D$ markers are being developed that reflect alterations of relevant pathways, and blood markers in MS hold the promise of being able to distinguish between inflammatory and neurodegenerative aspects of the disease.

Finally, this issue of Neurotherapeutics includes discussions of clinical outcome measures assessing function. Loss of strength has been recognized for years as being a critical cause of disability in many peripheral nerve diseases, as well as in motor neuron disease. However, quantitative methods that are reliable across study centers and evaluators have not been routinely employed. Recently, different methods of assessing strength have been implemented in clinical trials of ALS and spinal muscular atrophy with very encouraging results [17]. Similarly, while functional rating scales are commonly used as outcome measures in diseases as diverse as stroke, MS, AD, ALS, and Parkinson's disease, recent modifications have improved the metric qualities of these measures, and allowed for use in the home [18]. The recent development of patientreported outcome measures has also sparked new interest in the use of functional scales in a wide range of neurologic disease.

Overall, this issue demonstrates the wide range of new markers being developed in neurologic disease. The overall impression is that fields in which sophisticated markers have been developed are also the fields for which the most dramatic progress in new therapy has been made. Thus, the ongoing work highlighted here raises hope that fields where progress has not been so rapid may see this change, at least in part owing to better assessment.

We, the guest editors, would like to thank Linda Powell for helping us organize this issue, from identification of authors to encouraging timely submission and reviews, as well as the Editor in Chief of Neurotherapeutics, Dr. Maral Mouradian, for her encouragement and support of this effort. 
Required Author Forms Disclosure forms provided by the authors are available with the online version of this article.

\section{References}

1. Kumar DR, Yale SH, Mazza JH. Jean-Martin Charcot: the father of neurology. Clin Med Res 2011;9:46-49.

2. Maxwell SS. Is the conduction of the nerve impulse a chemical or physical process? J Biol Chem 1907;3:359-385.

3. Falco-Walter J, Owen C, Sharma M, Reggi C, Yu M, Stoub TR, Stein MA. Magnetoencephalography and new imaging modalities in epilepsy. Neurotherapeutics 2016.

4. Menke R, Agosta F, Grosskreutz J, Filippi M, Turner MA. Neuroimaging endpoints in amyotrophic lateral sclerosis. Neurotherapeutics 2016.

5. Fox RJ, Ontaneda D. Imaging as an outcome measure in multiple sclerosis. Neurotherapeutics 2016.

6. Counts SE, Ikonomovic MD, Mercado N, Vega IE, Mufson EJ. Biomarkers for the early detection and progression of Alzheimer's disease. Neurotherapeutics 2016.

7. Anand K, Sabbagh MN. Amyloid imaging: poised for integration into medical practice. Neurotherapeutics 2016.
8. Brosch JR, Farlow MR, Risacher SL, Apostolova LG, MD. Tau imaging in Alzheimer's disease diagnosis and clinical trials. Neurotherapeutics 2016.

9. McComas AJ, Fawcett PR, Campbell MJ, Sica RE. Electrophysiological estimation of the number of motor units within a human muscle. J Neurol Neurosurg Psychiatry 1971;34:121131

10. Henderson RD, McCombe P. Assessment of motor units in neuromuscular disease. Neurotherapeutics 2016.

11. Park S, Kiernan M, Vucic S. Axonal excitability in amyotrophic lateral sclerosis. Neurotherapeutics 2016.

12. Vucic S, Kiernan M. Transcranial magnetic stimulation for the assessment of neurodegenerative disease. Neurotherapeutics 2016.

13. Rutkove S, Sanchez B. Electrical impedance myography and its application in neuromuscular disease. Neurotherapeutics 2016.

14. Vu LT, Bowser RB. Fluid based biomarkers for amyotrophic lateral sclerosis. Neurotherapeutics 2016.

15. El Ayoubi NK, Khoury SJ. Blood biomarkers as outcome measures in inflammatory neurologic disease. Neurotherapeutics 2016.

16. Paganoni S. Urate as a marker of risk and progression of neurodegenerative disease. Neurotherapeutics 2016.

17. Shefner JM. Strength testing in motor neuron diseases. Neurotherapeutics 2016.

18. Narayanaswami P. The spectrum of functional rating scales in neurology clinical trials. Neurotherapeutics 2016. 